\title{
Upgrade of electronics of neutron monitors DOMC and DOMB
}

\author{
Stepan Poluianov 1,2, Ilya Usoskin ${ }^{1,2}$, Du Toit Strauss ${ }^{\circledR 3}$ \\ Correspondence \\ Sodankylä Geophysical Observatory, University of Oulu, Finland \\ stepan.poluianov@oulu.fi, ilya.usoskin@oulu.fi \\ ${ }^{2}$ Space Physics and Astronomy Research Unit, University of Oulu, Finland \\ ${ }^{3}$ Centre of Space Research, North-West University, Potchefstroom, South Africa \\ dutoit.strauss@nwu.ac.za
}

\section{OPEN ACCESS}

This work is published under the Creative Commons Attribution 4.0 International licence (CC BY 4.0)

Please note that individual, appropriately marked parts of the work may be excluded from the licence mentioned or may be subject to other copyright conditions. If such thirdparty materiat is not under the Creative Commons license any copying, editing or public reproduction is only permitted with the prior consent of the respective copy right owner or on the basis of relevant legal authorization regulations.

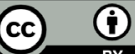

\section{Keywords}

cosmic rays; neutron monitor; data management

\begin{abstract}
DOMC and DOMB neutron monitors (NM) operate at the Concordia research station (Dome $C$ on the Antarctic plateau, $75^{\circ} 06^{\prime} \mathrm{S}, 123^{\circ} 23^{\prime} \mathrm{E}, 3233 \mathrm{~m}$ a.s.l.) since 2015 . Their high elevation and proximity to the geomagnetic pole provide low atmospheric and geomagnetic cutoffs and, therefore, the exceptionally high sensitivity to lowenergy cosmic rays. The instruments are the so-called mini neutron monitors with $\mathrm{BF}_{3}$-filled counter tubes. DOMC has the standard design with a lead neutron multiplier and DOMB is a so-called "bare" (lead-free) unit. We report on a recent upgrade of the electronics heads of these instruments. The new heads have a modular architecture, built upon a single-board computer Raspberry Pi. The upgrade increases the capabilities of the instruments in two aspects: (1) measurements, particularly, of cosmic ray multiplicity; (2) remote control and monitoring. The new electronic heads register each pulse from a detector, giving a timestamp with microsecond precision, which is crucial for multiplicity measurements. Many important parameters (e.g., high voltage, pulse detection thresholds) can be controlled and adjusted remotely with the new design. High computing power allows performing data processing on the fly. The upgrade increases the capability of DOMC and DOMB in cosmic ray measurements and improves control of the operation of the neutron monitors.
\end{abstract}

\section{Introduction}

DOMC and DOMB are two mini neutron monitors (NM) located at the polar, high-altitude Concordia research station (Dome C on the Antarctic plateau, $75^{\circ} 06^{\prime} \mathrm{S}, 123^{\circ} 23^{\prime} \mathrm{E}, 3233 \mathrm{~m}$ a.s.l.) - see figures 1 and 2. The instruments are described in detail in Poluianov et al. (2015). Both neutron monitors were built with the same design, however, there is a difference in the lead neutron producer layer. DOMC is standard (with lead) and DOMB is so-called "bare" (lead-free) instrument. In 2019, they got a significant upgrade with new electronics heads, providing new abilities in data analysis and instrument control (Strauss et al. 2020). 

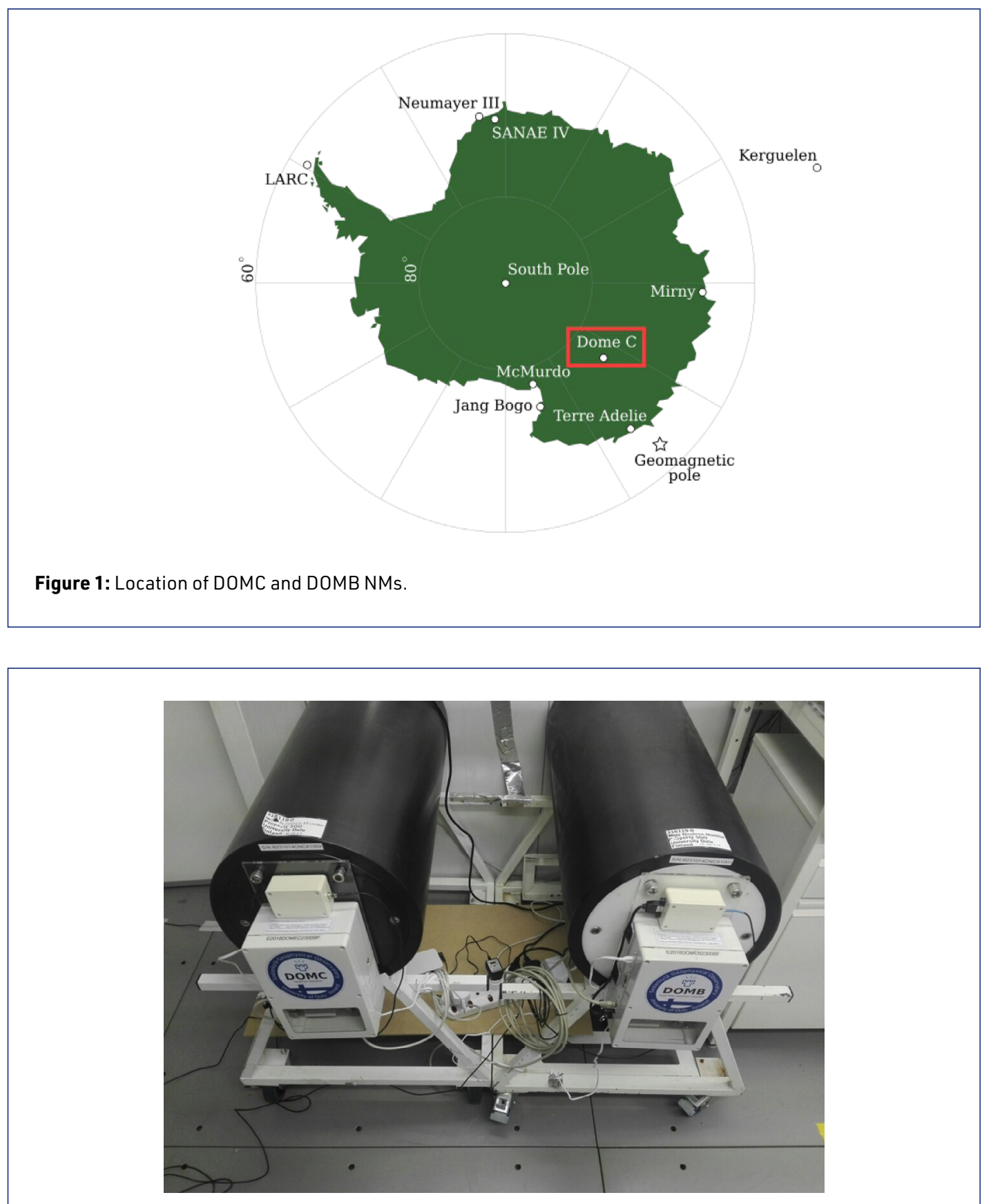

Figure 2: DOMC and DOMB NMs.

\section{New electronics heads}

The electronics heads (as well as the neutron monitors earlier) were made by the Centre for Space Research, North-West University (Potchefstroom, South Africa). Each head is built around a singleboard Raspberry Pi 3 B computer and has a modular design. There is an ADC board responsible for the digitization of pulses from the detector, an amplifier, a high-voltage module, pressure/temperature/humidity sensors, a GPS-module for time synchronization. 


\subsection{New features}

The new head is a fully-functional computer with GNU/Linux onboard, thus, it makes interactions between the instrument and other servers very easy. It is possible to operate the instrument via SSH and FTP/SFTP protocols, and therefore to use virtually any standard methods applicable to a GNU/Linux computer.

More important parameters (high voltage at the detector, pulse height and length discriminators) can be set up remotely. This is possible because all parts of the head, responsible for pulse handling and power supplying of the detector, are controlled by the central Raspberry Pi board.

GPS time synchronization is optional. The internal clock of the head can be set up with the network time protocol (NTP) or manually.

The new head registers each pulse from the detector individually: it records its timestamp, length, maximum magnitude and fully digitized time profile in volts (figure 3 ).

The pulse timestamp is recorded with the microsecond precision, while the earlier version of the electronics could record it with the millisecond resolution.

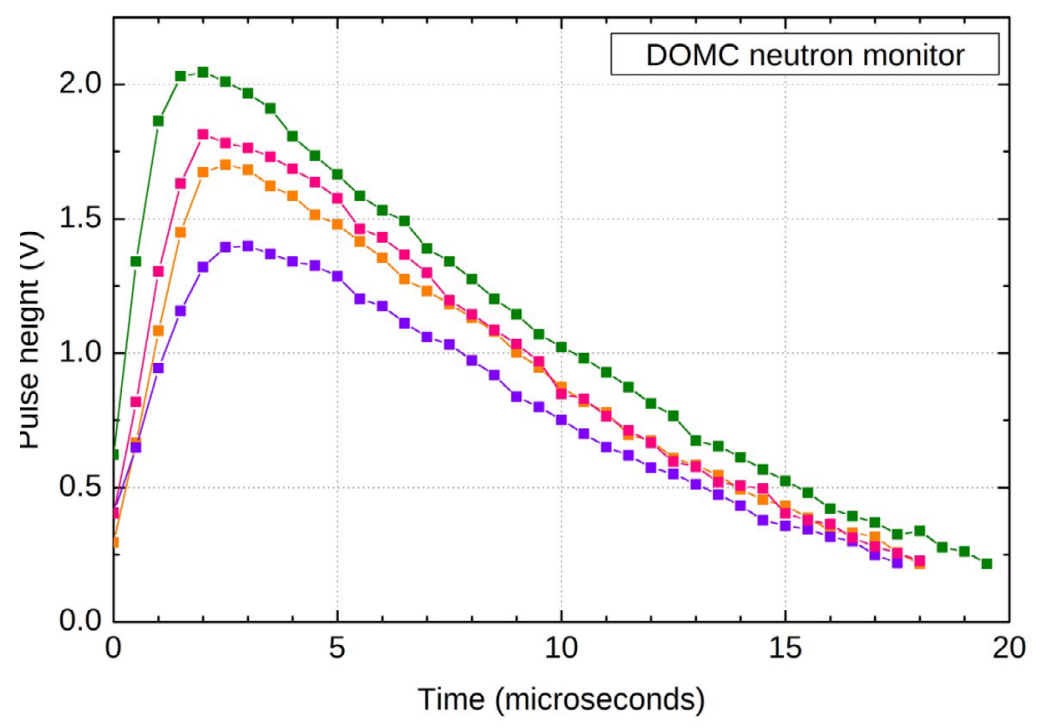

Figure 3: Examples of pulse time profiles.

\subsection{Data coming from DOMC and DOMB}

A new electronic head sends the following data: count data (timestamp, length, maximum magnitude and profile of every pulse), atmospheric pressure/temperature/humidity from two sensors, high voltage value, GPS coordinates, log data.

New features of the electronics lead to a significant increase of the amount of data produced by an instrument: for example, DOMC with the average count rate $1300 \mathrm{cts} / \mathrm{min}$ sends about $400 \mathrm{Mb} /$ day of raw uncompressed data. To handle increased volumes, the data management system at the Oulu cosmic ray station has been upgraded (see the proceedings Poluianov et al. "Data management at the Oulu cosmic ray station" at this symposium).

\subsection{New opportunities in the data processing}

The new electronics, thanks to its microsecond resolution in pulse timing, allow analyzing the cosmic ray multiplicity in DOMC and DOMB records. Pulse height/length discrimination is possible 
not only in real time by the head's ADC board but also retrospectively from raw data files (figure 4). It may be useful if the noise level changes. Pulse height histograms are also available and they are an important tool in monitoring the NM operation.

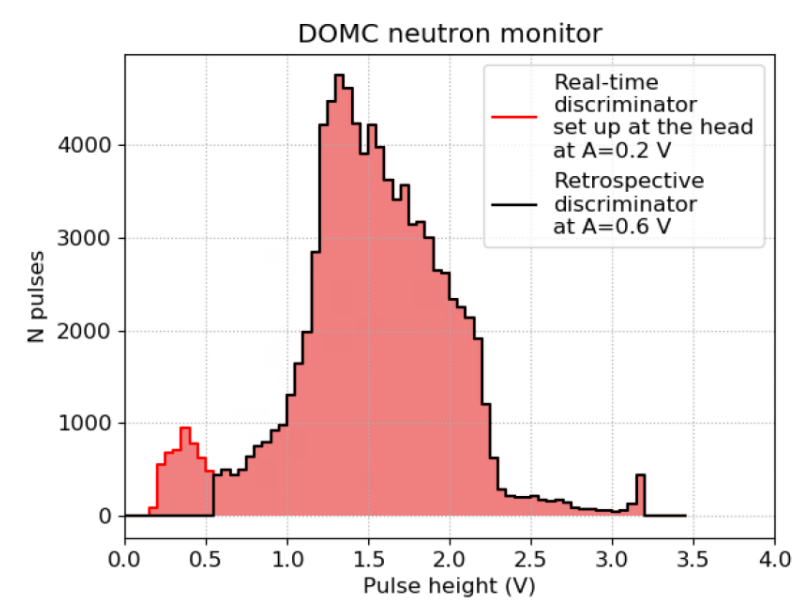

Figure 4: Pulse-height histogram from DOMC data (1 March 2020).

\subsection{New opportunities in remote control}

Since Raspberry Pi in the NM head is a fully-functional Linux computer, standard methods are applicable for its administration. This makes integration of the instrument to existing computer infrastructure very easy. At the Concordia station, DOMC and DOMB NMs are connected to the Concordia-NM server via local network. The server, in turn, sends data to the Oulu cosmic ray station via HERMES (a system for data transfer from Antarctica). The NM is fully controlled via SSH over the Concordia-NM server and Concordia VPN (figure 5).

It is also possible to remotely set up/adjust parameters important for CR measurements such as high-voltage, pulse height and length discriminations.

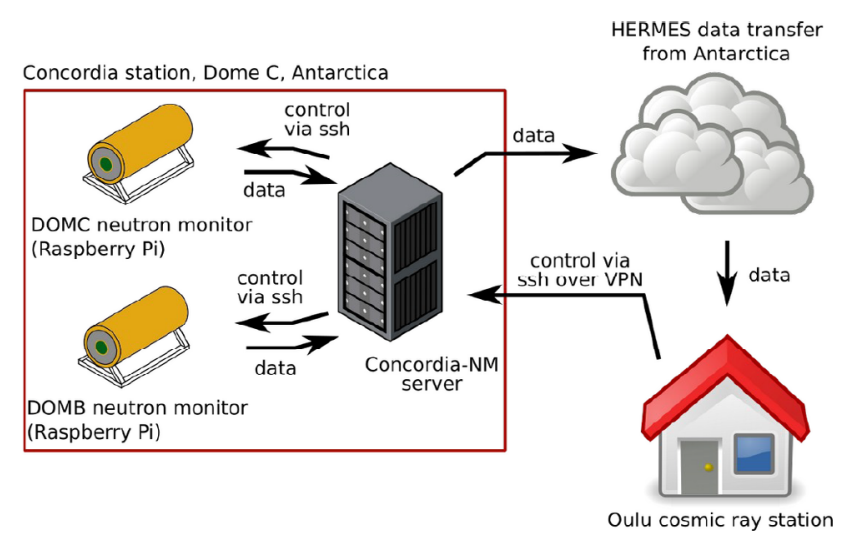

Figure 5: Dataflow of DOMC and DOMB NMs. 


\section{Summary}

DOMC and DOMB NM received new electronics in 2019. The upgraded instruments can be fully controlled remotely, which is a big advantage in operation in Antarctica. Registration of pulses with microsecond precision, as well as of their profiles, opens new opportunities in cosmic ray multiplicity measurements and fine control of the operation of the instruments.

\section{Acknowledgments}

The work was partly supported by FINNARP and Academy of Finland (projects 264378 CRIPA, 304435 CRIPA-X, ReSoLVE and 321882 ESPERA). We are thankful for the hospitality of the Italian Polar Programme PNRA (via the LTCPAA PNRA 2015/AC3 project) and the French Polar Institute IPEV.

\section{References}

Poluianov, S., Usoskin, I., Mishev, A., Moraal, H., Kruger, H., et al., 2015, Mini Neutron Monitors at Concordia Research Station, Central Antarctica, J. Astron. Space Sci., 32, 281-284, DOI: https://doi.org/10.5140/JASS.2015.32.4.281

Strauss, D. T., Poluianov, S., van der Merwe, C., Krueger, H., Diedericks, C., et al., 2020, The mini-neutron monitor: a new approach in neutron monitor design, J. Space Weather Space Clim., 10,39, DOI: https://dx.doi.org/10.1051/swsc/2020038 